Research Article

\title{
The Local and Parallel Finite Element Scheme for Electric Structure Eigenvalue Problems
}

\author{
Fubiao Lin $\mathbb{D D}^{1},{ }^{1}$ Junying Cao $\mathbb{D}^{2},{ }^{2}$ and Zhixin Liu $\mathbb{D}^{1,3}$ \\ ${ }^{1}$ School of Mathematics and Statistics, Guizhou University of Finance and Economics, Guiyang 550025, China \\ ${ }^{2}$ School of Data Science and Information Engineering, Guizhou Minzu University, Guiyang 550025, China \\ ${ }^{3}$ Guizhou Key Laboratory of Big Data Statistical Analysis, Guizhou University of Finance and Economics, Guiyang, \\ Guizhou 550025, China \\ Correspondence should be addressed to Zhixin Liu; xinxin905@126.com
}

Received 30 April 2021; Revised 26 June 2021; Accepted 25 July 2021; Published 20 August 2021

Academic Editor: Qiang Guo

Copyright (c) 2021 Fubiao Lin et al. This is an open access article distributed under the Creative Commons Attribution License, which permits unrestricted use, distribution, and reproduction in any medium, provided the original work is properly cited.

\begin{abstract}
In this paper, an efficient multiscale finite element method via local defect-correction technique is developed. This method is used to solve the Schrödinger eigenvalue problem with three-dimensional domain. First, this paper considers a three-dimensional bounded spherical region, which is the truncation of a three-dimensional unbounded region. Using polar coordinate transformation, we successfully transform the three-dimensional problem into a series of one-dimensional eigenvalue problems. These one-dimensional eigenvalue problems also bring singularity. Second, using local refinement technique, we establish a new multiscale finite element discretization method. The scheme can correct the defects repeatedly on the local refinement grid, which can solve the singularity problem efficiently. Finally, the error estimates of eigenvalues and eigenfunctions are also proved. Numerical examples show that our numerical method can significantly improve the accuracy of eigenvalues.
\end{abstract}

\section{Introduction}

As an important equation in quantum mechanics, Schrödinger eigenvalue problems have important physical background modern electronic structure computations $[1,2]$. Thus, finite element methods for solving this problem become an important topic which has attracted the attention of mathematical and physical fields: a priori error estimate is discussed in [3], some posteriori error estimates and adaptive algorithms have been studied in [4-7], and, in addition, it also includes twoscale method [8-12] and the extrapolation methods [13-16].

It is worth noting that some researchers $[5,6,17]$ constructed a series of efficient algorithms to solve PDE eigenvalue problems with angular singularity. For elliptic boundary value problem, Xu and Zhou [18] combined two-grid finite element discretization scheme with the local defect correction to propose a general and powerful parallel-computing technique. This technique has been used and developed by many scholars, for instance, it can be used to solve Stokes equation (see $[19,20])$, Especially, Xu and Zhou [21], Dai and Zhou [22], and Bi et al. [23-25] developed this method and established local and parallel three-scale finite element discretizations for symmetric elliptic singular eigenvalue problems.

As a matter of fact, due to the influence of Coulomb potentials, the convergence order of three-dimensional numerical methods and the computational efficiency of numerical methods will further deteriorate [26]. Therefore, one of the most direct and effective methods is to transform the three-dimensional problem into one-dimensional problem. Inspired by [27-29] and others references, it is necessary to further study the high-precision numerical method for singular problems. Therefore, in this paper, we turn to discuss finite element multiscale discretization based on local defect correction. We further apply local defectcorrection technique proposed by $\mathrm{Xu}$ and Zhou to Schrödinger eigenvalue problems, and our work has the following features. (1) We first extend local and parallel three-scale finite element discretizations for symmetric eigenvalue problems established by Dai and Zhou [22] to solve Schrödinger eigenvalue problem. (2) Based on [23], we establish a new multiscale finite element discretization method by local refinement, and this scheme repeatedly 
makes defect correction on finer and finer local meshes to make up for accuracy loss caused by abrupt changes of local mesh size in three-scale scheme. (3) For the two-scale algorithms in $[8,10]$, we prove the local error estimates of eigenfunctions. (4) Our scheme is simple and easy to carry out, and theoretical analysis and numerical experiment verify its efficiency to solve the singular Schrödinger eigenvalue problem.

The rest of this paper is organized in the following way. in Section 2, we will briefly introduce Schrödinger eigenvalue problem and the associated dimension reduction scheme. In Section 3, we will establish the multiscale finite element method. The error estimates of eigenvalues and eigenfunctions will be studied in Section 4. Several numerical experiments are presented in Section 5 to demonstrate the accuracy and efficiency of our algorithm. Some concluding remarks are given in Section 6.

$$
\begin{aligned}
-\frac{1}{2} \Delta \psi+V \psi & =\lambda \psi, \quad \text { in } \mathbb{R}^{3}, \\
\lim _{|x| \rightarrow \infty} \psi & =0,
\end{aligned}
$$

where $V$ is the effective potential and $x=\left(x_{1}, x_{2}, x_{3}\right) \in \mathbb{R}^{3}$.

Applying a truncation from a unbounded domain $\mathbb{R}^{3}$ to a bounded spherical domain $\mathbb{B}^{3}:=\left\{x \in \mathbb{R}^{3}:|x|<R\right\}$, we find

$$
\begin{array}{r}
-\frac{1}{2} \Delta \psi+V \psi=\lambda \psi, \quad \text { in } \mathbb{B}^{3}, \\
\psi=0, \quad \text { on } \partial \mathbb{B}^{3} .
\end{array}
$$

Using the spherical coordinate transformation [27], problems (1) and (2) are equivalent to

\section{Dimension Reduction Scheme}

Consider the Schrödinger eigenvalue problem:

$$
\begin{aligned}
-\frac{1}{2} \partial_{t}\left((t+1)^{2} \partial_{t} u_{k}\right)+\frac{k(k+1)}{2} u_{k}+\frac{R^{2}}{4}(t+1)^{2} V_{0}(r) u_{k} & =\lambda_{k} \frac{R^{2}}{4}(t+1)^{2} u_{k}, \\
u_{k}(1) & =0 .
\end{aligned}
$$

This problem has singularities towards $t=-1$.

Next, we introduce the weighted Sobolev spaces on $\Omega:=(-1,1)$ :

$$
\begin{aligned}
L_{\omega}^{2}(\Omega) & :=\left\{v: \int_{\Omega} \omega v^{2} \mathrm{~d} t<\infty\right\}, \\
H_{\omega, k}^{1}(\Omega) & :=\left\{v: \partial_{t}^{m} v \in L_{\omega}^{2}(\Omega) \text { if } k=0, \partial_{t}^{m} v \in L_{\omega^{m}}^{2}(\Omega) \text { if } k\right. \\
& \geq 1, m=0,1, v(1)=0\},
\end{aligned}
$$

with $\omega(t):=(t+1)^{2}$. For simplicity of notations in the reminder, we omit the subscript $k$ in $u_{k}$ and $\lambda_{k}$ and denote $H_{\omega, k}^{1}(\Omega)$ by $H_{\omega}^{1}(\Omega)$ for short.

The variational form of (4) and (5) is to find $\lambda \in \mathbb{R}$ and nonzero $u \in H_{\omega}^{1}(\Omega)$, satisfying

$$
a(u, v)=\lambda b(u, v), \quad \forall v \in H_{\omega}^{1}(\Omega),
$$

where

$$
\begin{aligned}
a(u, v)= & \int_{\Omega} \frac{1}{2}(t+1)^{2} u^{\prime} v^{\prime}+\frac{k(k+1)}{2} u v+\frac{R^{2}}{4}(t+1)^{2} \\
& \left(V_{0}(r)+\mu\right) u v \mathrm{~d} t, \\
b(u, v)= & \int_{\Omega} \frac{R^{2}}{4}(t+1)^{2} u v \mathrm{~d} t,
\end{aligned}
$$

with $\mu>0$. According to Theorem 1 in [27], $a(\cdot, \cdot)$ and $b(\cdot, \cdot)$ define the inner product in $H_{\omega}^{1}(\Omega)$ and $L_{\omega}^{2}(\Omega)$, respectively. Let $\|\cdot\|_{a}$ and $\|\cdot\|_{b}$ be the norm induced by the inner products $a(\cdot, \cdot)$ and $b(\cdot, \cdot)$. Let $\|\cdot\|_{a}$ and $\|\cdot\|_{b}$.

For $D \subset \Omega_{0} \subset \Omega$, we use $D \subset \subset \Omega_{0}$ to mean that $\operatorname{dist}\left(\partial D \backslash \partial \Omega, \partial \Omega_{0} \backslash \partial \Omega\right)>0$.

Assume that $\pi_{h}(\Omega)=\{\tau\}$ is a mesh of $\Omega$ with mesh-size function $h(x)$ whose value is the diameter $h_{\tau}$ of the element $\tau$ containing $x$, and $h(\Omega)=\max _{x \in \Omega} h(x)$ is the mesh diameter of $\pi_{h}(\Omega)$. We write $h(\Omega)$ as $h$ for simplicity. Let $V_{h}(\Omega) \subset C(\bar{\Omega})$, defined on $\pi_{h}(\Omega)$, be a space of piecewise polynomials, and $V_{h}^{0}(\Omega)=V_{h}(\Omega) \cap H_{\omega}^{1}(\Omega)$. Given $G \subset \Omega$, we define $\pi_{h}(G)$ and $V_{h}(G)$ to be the restriction of $\pi_{h}(\Omega)$ and $V_{h}(\Omega)$ to $G$, respectively, and

$$
\begin{aligned}
& V_{h}^{0}(G)=\left\{v \in V_{h}^{0}(\Omega):\left.v\right|_{\partial G \backslash \partial \Omega}=0\right\}, \\
& V_{0}^{h}(G)=\left\{v \in V_{h}^{0}(\Omega): \operatorname{supp} v \backslash \partial \Omega \subset \subset G\right\} .
\end{aligned}
$$

For any $G \subset \Omega$ mentioned in this paper, we assume that it aligns with $\pi_{h}(\Omega)$ when necessary.

In this part, $C$ denotes a positive constant independent of $h$, which may not be the same constant in different places. For simplicity, we use the symbol $x \lesssim y$ to mean that $x \leq C y$.

We adopt the following assumptions similar as in [18] for meshes and finite element space.

(A0) There exists $v \geq 1$ such that $h(\Omega)^{v} \lesssim h(x), \forall x \in \Omega$. 
(A1) There exists $r \geq 1$ such that, for $w \in H_{\omega}^{1}(\Omega)$ $\cap H^{1+t}(\Omega)$,

$\inf _{v \in V_{h}^{0}(\Omega)}\left(\left\|h^{-1}(w-v)\right\|_{b}+\|w-v\|_{a}\right) \leq h^{t}\|w\|_{1+t}, \quad 0 \leq t \leq r$.

(A2) Inverse Estimate. For any $v \in V_{h}\left(\Omega_{1}\right)$, $\|v\|_{a, \Omega_{1}} \leqslant\left\|h^{-1} v\right\|_{b, \Omega_{1}}$.

(A3) Superapproximation. For $G \subset \Omega_{1}$, let $\widetilde{\omega} \in C^{\infty}(\bar{\Omega})$ with supp $\widetilde{\omega} \backslash \partial \Omega \subset \subset G$. Then, for any $w \in V_{h}(G)$, there exists $v \in V_{h}^{0}(G)$ such that $\left\|h^{-1}(\widetilde{\omega} w-v)\right\|_{a, G}$ $\leqslant\|w\|_{a, G}$.

The finite element approximation of (7) is given; find $\lambda_{h} \in C$ and $u_{h} \in V_{h}^{0}(\Omega),\left\|u_{h}\right\|_{a}=1$, satisfying

$$
a\left(u_{h}, v\right)=\lambda_{h} b\left(u_{h}, v\right), \quad \forall v \in V_{h}^{0}(\Omega) .
$$

Define the solution operator $T: L_{\omega}^{2}(\Omega) \longrightarrow H_{\omega}^{1}(\Omega)$ and $T_{h}: L_{\omega}^{2}(\Omega) \longrightarrow V_{h}^{0}(\Omega)$ as follows:

$$
\begin{gathered}
a(T g, v)=b(g, v), \quad \forall v \in H_{\omega}^{1}(\Omega), \\
a\left(T_{h} g, v\right)=b(g, v), \quad \forall v \in V_{h}^{0}(\Omega) .
\end{gathered}
$$

Problems (7) and (11) have the equivalent operator forms (14) and (15), respectively:

$$
\begin{aligned}
T u & =\lambda^{-1} u, \\
T_{h} u_{h} & =\lambda_{h}^{-1} u_{h} .
\end{aligned}
$$

The following regularity assumption is needed in theoretical analysis. For any $f \in L_{\omega}^{2}(\Omega), T f \in H_{\omega}^{1}(\Omega) \cap H^{1+\gamma}$ $(\Omega)$ satisfies

$$
\|T f\|_{1+\gamma} \leq C_{\Omega}\|f\|_{b} .
$$

According to [30] and Section 5.5 in [31], the above assumption is reasonable.

For some $G \subset \Omega$, we need the following local regularity assumption.

$\mathbf{R}(\mathbf{G})$ : for any $f \in L_{\omega}^{2}(G)$, there exists a $\phi \in H_{\omega}^{1}(G)$ $\cap H^{1+\gamma}(G)$ satisfying

$$
\begin{aligned}
a(\phi, v) & =b(f, v), \quad \forall v \in H_{\omega}^{1}(G), \\
\|\phi\|_{1+\gamma, G} & \leq C_{G}\|f\|_{b, G},
\end{aligned}
$$

where $C_{\Omega}$ and $C_{G}$ are two priori constants.

Define the Ritz projection $P_{h}: H_{\omega}^{1}(\Omega) \longrightarrow V_{h}^{0}(\Omega)$ by

$$
a\left(u-P_{h} u, v\right)=0, \quad \forall v \in V_{h}^{0}(\Omega) .
$$

Then, $T_{h}=P_{h} T$ (see [32]).

Let $M(\lambda)$ be the space spanned by all generalized eigenfunctions corresponding to $\lambda$ of $T, M_{h}(\lambda)$ be the space spanned by all generalized eigenfunctions corresponding to all eigenvalues of $T_{h}$ that converge to $\lambda$.

We also need the lemma as follows (see $[8,10])$.
Lemma 1. Let $(\lambda, u)$ be an eigenpair of (7). Then, for all $w \in H_{0}^{1}(\Omega), w \neq 0$,

$$
\frac{a(w, w)}{b(w, w)}-\lambda=\frac{a(w-u, w-u)}{b(w, w)}-\lambda \frac{b(w-u, w-u)}{b(w, w)} .
$$

The a priori error estimates of the finite element approximations (11) can be found in $[3,32]$.

Lemma 2. Assume that $M(\lambda) \subset H^{r+s}(\Omega)(0<s<1)$. Then,

$$
\left|\lambda_{h}-\lambda\right| \lesssim h^{2 r+2 s-2},
$$

and let $u_{h} \in M_{h}(\lambda)$ with $\left\|u_{h}\right\|_{b}=1$; then, there is $u \in M(\lambda)$ such that

$$
\begin{aligned}
& \left\|u_{h}-u\right\|_{a} \leqslant h^{r+s-1}, \\
& \left\|u_{h}-u\right\|_{b} \leqslant h^{r+s-1+\gamma} .
\end{aligned}
$$

The authors in $[18,33]$ studied the local behavior of finite element. The following results are given in [18].

Lemma 3. Suppose that $f \in L_{\omega}^{2}(\Omega)$ and $G \subset \subset \Omega_{0} \subset \Omega$. If $w \in V_{h}\left(\Omega_{0}\right)$ satisfies

$$
a(w, v)=b(f, v), \quad \forall v \in V_{0}^{h}\left(\Omega_{0}\right),
$$

then

$$
\|w\|_{a, G} \lesssim\|w\|_{b, \Omega_{0}}+\|f\|_{b, \Omega_{0}} .
$$

Proof. Let $p \geq 2 v-1$ be an integer, and let

$$
D \subset \subset \Omega_{p} \subset \subset \Omega_{p-1} \subset \subset \cdots \subset \subset \Omega_{1} \subset \subset \Omega_{0} .
$$

Choose $D_{1} \subset \Omega$ satisfying $D \subset \subset D_{1} \subset \subset \Omega_{p}$ and $\widetilde{\omega} \in C^{\infty}(\bar{\Omega})$ such that supp $\widetilde{\omega} \subset \subset \Omega_{p}$ and $\widetilde{\omega} \equiv 1$ on $\overline{D_{1}}$. Then, from (A3), there exists $v \in V_{0}^{h}\left(\Omega_{p}\right)$ such that

$$
\left\|\widetilde{\omega}^{2} w-v\right\|_{a, \Omega_{p}} \leq h_{\Omega_{0}}\|w\|_{a, \Omega_{p}}
$$

so we have

$$
\begin{gathered}
a\left(w, \widetilde{\omega}^{2} w-v\right) \leq h_{\Omega_{0}}\|w\|_{a, \Omega_{p}}^{2}, \\
|b(f, v)| \leq\|f\|_{b, \Omega_{0}}\|v\|_{b, \Omega_{p}} \leq\|f\|_{b, \Omega_{0}}\left(h_{\Omega_{0}}\|w\|_{a, \Omega_{p}}+\|\tilde{\omega} w\|_{a, \Omega}\right) .
\end{gathered}
$$

Since $v \in V_{0}^{h}\left(\Omega_{p}\right) \subset V_{0}^{h}\left(\Omega_{0}\right)$, the definition $w$ implies

$$
a\left(w, \widetilde{\omega}^{2} w\right)=a\left(w, \widetilde{\omega}^{2} w-v\right)+b(f, v) .
$$

A simple calculation shows that

$a(\widetilde{\omega} w, \widetilde{\omega} w) \lesssim a\left(w, \widetilde{\omega}^{2} w\right)+\|w\|_{b, \Omega_{0}}^{2}, \quad \forall w \in H_{\omega}^{1}(\Omega)$.

It follows from (26)-(29) that 


$$
\begin{aligned}
\|\widetilde{\omega} w\|_{a, \Omega}^{2} & \leqslant a\left(w, \widetilde{\omega}^{2} w\right)+\|w\|_{b, \Omega_{0}}^{2} \\
& =a\left(w, \widetilde{\omega}^{2} w-v\right)+\|w\|_{b, \Omega_{0}}^{2}+b(f, v) \\
& \leq h_{\Omega_{0}}\|w\|_{a, \Omega_{p}}^{2}+\|w\|_{b, \Omega_{0}}^{2}+\|f\|_{b, \Omega_{0}}\left(h_{\Omega_{0}}\|w\|_{1, \Omega_{p}}+\|\widetilde{\omega} w\|_{a, \Omega}\right),
\end{aligned}
$$

and thus,

$$
\|w\|_{a, D} \lesssim h_{\Omega_{0}}^{1 / 2}\|w\|_{a, \Omega_{p}}+\|w\|_{b, \Omega_{0}}+\|f\|_{b, \Omega_{0}} .
$$

Similarly, we can obtain

$$
\|w\|_{a, \Omega_{j}} \leqslant h_{\Omega_{0}}^{1 / 2}\|w\|_{a, \Omega_{j-1}}+\|w\|_{b, \Omega_{0}}+\|f\|_{b, \Omega_{0}}, \quad j=1,2, \ldots, p .
$$

By using (31) and (32), we get from (A0) and (A2) and inverse estimate that

$$
\begin{aligned}
\|w\|_{a, D} & \leq h_{\Omega_{0}}^{(p+1) / 2}\|w\|_{a, \Omega_{0}}+\|w\|_{b, \Omega_{0}}+\|f\|_{b, \Omega_{0}} \\
& \leq h_{\Omega_{0}}^{(p+1) / 2}\left\|h^{-1} w\right\|_{b, \Omega_{0}}+\|w\|_{b, \Omega_{0}}+\|f\|_{b, \Omega_{0}} \\
& \leq\|w\|_{b, \Omega_{0}}+\|f\|_{b, \Omega_{0}} .
\end{aligned}
$$

This completes the proof.

Lemma 4. Suppose that $G \subset \subset \Omega_{0} \subset \Omega$. Then, the following estimates are valid:

$$
\begin{gathered}
h^{\gamma}\left\|u-P_{h} u\right\|_{a, \Omega}+\left\|u-P_{h} u\right\|_{b, \Omega} \lesssim h^{\gamma} \inf _{v \in V_{h}^{0}(\Omega)}\|u-v\|_{a, \Omega}, \\
\left\|u-P_{h} u\right\|_{a, G} \lesssim \inf _{v \in V_{h}^{0}(\Omega)}\|u-v\|_{a, \Omega_{0}}+h^{\gamma}\left\|u-P_{h} u\right\|_{a, \Omega} .
\end{gathered}
$$

Proof. By (15), we obtain

$$
\left\|T_{h}\left(\lambda_{h} u_{h}-\lambda u\right)\right\|_{a} \lesssim\left\|\lambda_{h} u_{h}-\lambda u\right\|_{b} .
$$

By the definitions of $T, T_{h}$, and $P_{h}$, we deduce that

$$
\begin{aligned}
\lambda T u & =u, \\
\lambda T_{h} u & =P_{h} u .
\end{aligned}
$$
then,

Let $P_{h}^{\Omega_{0}}$ be the finite element projection onto $V_{0}^{h}\left(\Omega_{0}\right)$;

$$
a\left(P_{h} u-P_{h}^{\Omega_{0}} u, v\right)=0, \quad \forall v \in V_{0}^{h}\left(\Omega_{0}\right) .
$$

According to Lemma 3, we have

$$
\left\|P_{h} u-P_{h}^{\Omega_{0}} u\right\|_{a, G} \leqslant\left\|P_{h} u-P_{h}^{\Omega_{0}} u\right\|_{b, \Omega_{0}} .
$$

Then, by using (14) and (39), we conclude that

$$
\begin{aligned}
\left\|u-P_{h} u\right\|_{a, D} & \lesssim\left\|u-P_{h}^{\Omega_{0}} u+P_{h}^{\Omega_{0}} u-P_{h} u\right\|_{a, D} \\
& \lesssim \inf _{v \in V_{h}^{0}(\Omega)}\|u-v\|_{a, \Omega_{0}}+\left\|u-P_{h} u\right\|_{b, \Omega} .
\end{aligned}
$$

Thus, we derive (35) from (39).

\section{Multiscale Discretizations Based on Local Defect Correction}

Consider the eigenvalue problem (7) which has an isolated singular point $t=-1$ (e.g., see Figure 1).

Let $D \subset \subset \Omega$ be a given subdomain containing the singular point $z$, and we introduce domains:

$$
\Omega \supset \Omega_{1} \supset \Omega_{2} \supset \cdots \supset \Omega_{l} \supset \supset D .
$$

Let $\pi_{H}(\Omega)$ be a shape-regular grid, which is made up of simplices, with size $H \in(0,1), \pi_{w}(\Omega)$ be a refined mesoscopic shape-regular grid (from $\pi_{H}(\Omega)$ ), and $\pi_{h}\left(\Omega_{i}\right)$ be a locally refined grid (from $\pi_{h_{i-1}}\left(\Omega_{i-1}\right)$ ) that satisfies $h_{-1}=H$, $h_{0}=w, h_{i} \ll h_{i-1}(i=0,1, \ldots, l)$ (Figure 1 shows $\pi_{H}(\Omega)$, $\pi_{w}(\Omega)$, and $\pi_{h_{1}}\left(\Omega_{1}\right)$. Let $V_{H}^{0}(\Omega), V_{w}^{0}(\Omega)$, and $\left\{V_{h_{i}}^{0}\left(\Omega_{i}\right)\right\}_{1}$ be finite element spaces of degree less than or equal to $r$ defined on $\pi_{H}(\Omega), \pi_{w}(\Omega)$, and $\left\{\pi_{h_{i}}\left(\Omega_{i}\right)\right\}_{1}^{l}$, respectively.

Based on algorithm $B_{0}$ in [22], we establish the following three-scale discretization scheme.

Scheme 1. (three-scale discretizations based on local defect correction).

Step 1: solve (7) on a globally coarse grid $\pi_{H}(\Omega)$; find $\lambda_{H} \in \mathscr{C}, u_{H} \in V_{H}^{0}(\Omega)$ such that $\left\|u_{H}\right\|_{0}=1$ and

$$
a\left(u_{H}, v\right)=\lambda_{H} b\left(u_{H}, v\right), \quad \forall v \in V_{H}^{0}(\Omega) .
$$

Step 2: solve two linear boundary value problems on a globally mesoscopic grid $\pi_{w}(\Omega)$; find $u^{w} \in V_{w}^{0}(\Omega)$ such that

$$
a\left(u^{w}, v\right)=\lambda_{H} b\left(u_{H}, v\right), \quad \forall v \in V_{w}^{0}(\Omega),
$$

and then, compute the Rayleigh quotient $\lambda^{w}=a\left(u^{w}\right.$, $\left.u^{w}\right) / b\left(u^{w}, u^{w}\right)$.

Step 3: solve two linear boundary value problems on a locally fine grid $\pi_{h_{1}}\left(\Omega_{1}\right)$; find $e^{h_{1}} \in V_{h_{1}}^{0}\left(\Omega_{1}\right)$ such that

$$
a\left(e^{h_{1}}, v\right)=\lambda^{w} b\left(u^{w}, v\right)-a\left(u^{w}, v\right), \quad \forall v \in V_{h_{1}}^{0}\left(\Omega_{1}\right) \text {. }
$$

Step 4: set

$$
u^{w, h_{1}}= \begin{cases}u^{w}+e^{h_{1}}, & \text { on } \bar{\Omega}_{1}, \\ u^{w}, & \text { in } \Omega \backslash \bar{\Omega}_{1},\end{cases}
$$

and compute the Rayleigh quotient:

$$
\lambda^{w, h_{1}}=\frac{a\left(u^{w, h_{1}}, u^{w, h_{1}}\right)}{b\left(u^{w, h_{1}}, u^{w, h_{1}}\right)} .
$$

We use $\left(\lambda^{w, h_{1}}, u^{w, h_{1}}\right)$ obtained by Scheme 1 as the approximate eigenpair of (7). 

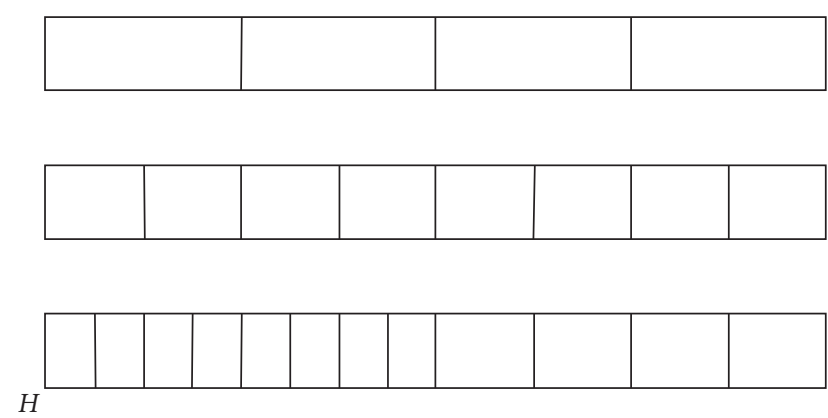

Figure 1: Finite element meshes.

It is obvious that $\left(\lambda^{w}, u^{w}\right)$ in Scheme 1 can be viewed as approximate eigenpairs obtained by the two-grid discretization scheme in $[8,10]$ from $\pi_{H}(\Omega)$ and $\pi_{w}(\Omega)$.

Using Scheme 1, abrupt changes of mesh size will appear near $\partial \Omega_{1}$. Influenced by the technique on the transition layer proposed by [23], we repeatedly use the local defect-correction technique to establish the following multiscale discretization scheme.

Scheme 2. (multiscale discretizations based on local defect correction).

Step 1: the same as that of Step 1 of Scheme 1.

Step 2: the same as that of Step 2 of Scheme 1.

Step 3: $u^{w, h_{0}} \Leftarrow \mathcal{u}^{w}$ and $\lambda^{w, h_{0}} \Leftarrow \lambda^{w}$.

Step 4: for $i=1,2, \ldots, l$, execute Step 5 and Step 6 .

Step 5: solve linear boundary value problems on locally fine grid $\pi_{h_{i}}\left(\Omega_{i}\right)$; find $e^{h_{i}} \in V_{h_{i}}^{0}\left(\Omega_{i}\right)$ such that

$a\left(e^{h_{i}}, v\right)=\lambda^{w, h_{i-1}} b\left(u^{w, h_{i-1}}, v\right)-a\left(u^{w, h_{i-1}}, v\right), \quad \forall v \in V_{h_{i}}^{0}\left(\Omega_{i}\right)$.

Step 6: set

$$
u^{w, h_{i}}= \begin{cases}u^{w, h_{i-1}}+e^{h_{i}}, & \text { on } \bar{\Omega}_{i}, \\ u^{w, h_{i-1}}, & \text { in } \Omega \backslash \bar{\Omega}_{i},\end{cases}
$$

and compute

$$
\lambda^{w, h_{i}}=\frac{a\left(u^{w, h_{i}}, u^{w, h_{i}}\right)}{b\left(u^{w, h_{i}}, u^{w, h_{i}}\right)} .
$$

We use $\left(\lambda^{w, h_{l}}, u^{w, h_{l}}\right)$ obtained by Scheme 2 as the approximate eigenpair of (7).

\section{Theoretical Analysis}

Next, we shall discuss the error estimates of Schemes 1 and 2. In our analysis, we introduce an auxiliary grid $\pi_{h_{i}}(\Omega)$ which is defined globally and denote the corresponding finite element space of degree $\leq r$ by $V_{h}^{0}(\Omega)(i=1,2, \ldots, l)$. We also assume that $\pi_{h_{i}}\left(\Omega_{i}\right)$ and $V_{h_{i}}^{0}\left(\Omega_{i}\right)$ are the restrictions of $\pi_{h_{i}}(\Omega)$ and a subspace of $V_{h_{i}}^{0_{i}}(\Omega)$ to $\Omega_{i}$, respectively, and

$$
V_{H}^{0}(\Omega) \subset V_{w}^{0}(\Omega) \subset V_{h_{1}}^{0}(\Omega) \subset V_{h_{2}}^{0}(\Omega) \subset \cdots \subset V_{h_{l}}^{0}(\Omega) .
$$

For $D$ and $\Omega_{i}$ stated at the beginning of Section 3, let $G_{i} \subset \Omega$ and $F \subset \Omega$ satisfy $D \subset F \subset \subset G_{i} \subset \subset \Omega_{i}(i=1,2$, $\ldots, l)$.

Theorem 1. Assume that $M(\lambda) \subset H^{r+s}(\Omega) \cap H^{r+1}(\Omega / \bar{D})$ and $(1<r+s, 0 \leq s<1)$, and $H$ is properly small. Then, there exists $u \in M(\lambda)$ such that

$$
\begin{gathered}
\left\|u^{w}-u\right\|_{a} \leq H^{r+s-1+\gamma}+w^{r+s-1}, \\
\left\|u^{w}-u\right\|_{b} \leq H^{r+s-1+\gamma}, \\
\left\|u^{w}-u\right\|_{a, \Omega \backslash \bar{F}} \leq H^{r+s-1+\gamma}+w^{r}, \\
\left|\lambda^{w}-\lambda\right| \leq H^{2 r+2 s-2+2 \gamma}+w^{2 r+2 s-2} .
\end{gathered}
$$

Proof. Let $u \in M(\lambda)$ such that $u-u_{H}$ satisfies Lemma 2 . From (12) and (13), Step 2 of Scheme 1, (14), and Lemmas 2 and 4 , we derive that

$$
\begin{aligned}
\left\|u^{w}-u\right\|_{a} & =\left\|\lambda_{H} T_{w} u_{H}-\lambda T u\right\|_{a} \\
& \leq\left\|\lambda_{H} T_{w} u_{H}-\lambda T_{w} u\right\|_{a}+\left\|\lambda T_{w} u-\lambda T u\right\|_{a} \\
& \leqslant\left\|\lambda_{H} u_{H}-\lambda u\right\|_{b}+\lambda\left\|P_{w} T u-T u\right\|_{a} \\
& \leqslant H^{r+s-1+\gamma}+w^{r+s-1},
\end{aligned}
$$

and then, (51) follows. By Lemmas 2 and 4,

$$
\begin{aligned}
\left\|u^{w}-u\right\|_{a, \Omega / \bar{D}} & \lessgtr\left\|\lambda_{H} u_{H}-\lambda u\right\|_{b}+\lambda\left\|P_{w} T u-T u\right\|_{a, \Omega / \bar{D}} \\
& \lesssim H^{r+s-1+\gamma}+w^{r},
\end{aligned}
$$

and then, (53) follows. By calculation,

$$
\begin{aligned}
\left\|u^{w}-u\right\|_{b} & =\left\|\lambda_{H} T_{w} u_{H}-\lambda T u\right\|_{b} \\
& \leq\left\|\lambda_{H} T_{w} u_{H}-\lambda T_{w} u\right\|_{b}+\left\|\lambda T_{w} u-\lambda T u\right\|_{b} \\
& \leq\left\|\lambda_{H} u_{H}-\lambda u\right\|_{b}+\lambda\left\|P_{w} T u-T u\right\|_{b} \\
& \leq H^{r+s-1+\gamma}+w^{r+s-1+\gamma} \\
& \leq H^{r+s-1+\gamma}
\end{aligned}
$$

and then, (52) follows. From (19), we have

$$
\lambda^{w}-\lambda=\frac{a\left(u^{w}-u, u^{w}-u\right)}{b\left(u^{w}, u^{w}\right)}-\lambda \frac{b\left(u^{w}-u, u^{w}-u\right)}{b\left(u^{w}, u^{w}\right)} .
$$

Note that $u_{H}$ and $u^{w}$ just approximate the same eigenfuntion $u$. The combination of (51), (52), and (58) yields (54).

Theorem 2 is a critical result in this paper, which develops the results of Theorem 3.3 in [22].

Theorem 2. Assume that $R\left(\Omega_{i}\right)$ holds $(i=1,2, \ldots, l)$, $u \in M(\lambda)$. Then, 


$$
\begin{aligned}
\left\|u^{w, h_{l}}-P_{h_{l}} u\right\|_{a} \leq & \left\|u-P_{h_{l}} u\right\|_{b, \Omega_{l}}+h_{l-1}^{\gamma}\left\|P_{h_{l}} u-u^{w, h_{l-1}}\right\|_{a, \Omega_{l}} \\
& +\left\|\lambda u-\lambda^{w, h_{l-2}} u^{w, h_{l-2}}\right\|_{b, \Omega_{l}}+\left\|\lambda^{w, h_{l-1}} u^{w, h_{l-1}}-\lambda u\right\|_{b} \\
& +\left\|u^{w, h_{l-1}}-P_{h_{l}} u\right\|_{a, \Omega \mid \bar{G}_{l}}+\left\|u^{w, h_{l-1}}-u\right\|_{a, \Omega_{l} \mid \bar{F}}, \quad l \geq 1 .
\end{aligned}
$$

Proof. Due to the inequality

$$
\begin{gathered}
\left\|u^{w, h_{l}}-P_{h_{l}} u\right\|_{a, \Omega} \leq\left\|u^{w, h_{l}}-P_{h_{l}} u\right\|_{a, D}+\left\|u^{w, h_{l}}-P_{h_{l}} u\right\|_{a, G_{l} \backslash \bar{D}} \\
+\left\|u^{w, h_{l}}-P_{h_{l}} u\right\|_{a, \Omega \backslash \overline{G_{l}}}
\end{gathered}
$$

we shall estimate $\left\|u^{w, h_{l}}-P_{h_{l}} u\right\|_{a, D},\left\|u^{w, h_{l}}-P_{h_{l}} u\right\|_{a, G_{l} \mid \bar{D}}$, and $\left\|u^{w, h_{l}}-P_{h_{l}} u\right\|_{a, \Omega \mid \overline{G_{l}}}$, respectively.

First, we proceed to estimate $\left\|u^{w, h_{l}}-P_{h_{l}} u\right\|_{a, D}$. From (18), (47), and (48), we derive

$$
\begin{aligned}
a\left(u^{w, h_{l}}-P_{h_{l}} u, v\right) & =a\left(u^{w, h_{l}}, v\right)-a\left(P_{h_{l}} u, v\right) \\
& =a\left(u^{w, h_{l-1}}+e^{h_{l}}, v\right)-a(u, v) \\
& =\lambda^{w, h_{l-1}} b\left(u^{w, h_{l-1}}, v\right)-\lambda b(u, v), \quad \forall v \in V_{h_{l}}^{0}\left(\Omega_{l}\right) .
\end{aligned}
$$

It is obvious that

$$
\begin{aligned}
& \lambda^{w, h_{l-1}} b\left(u^{w, h_{l-1}}, v\right)-\lambda b(u, v) \\
= & \left(\lambda^{w, h_{l-1}}-\lambda\right) b(u, v)+\lambda^{w, h_{l-1}} b\left(u^{w, h_{l-1}}-u, v\right), \quad \forall v \in H_{0}^{1}(\Omega),
\end{aligned}
$$

which together with (61) yields

$$
\begin{aligned}
a\left(u^{w, h_{l}}-P_{h_{l}} u, v\right)= & \left(\lambda^{w, h_{l-1}}-\lambda\right) b(u, v) \\
& +\lambda^{w, h_{l-1}} b\left(u^{w, h_{l-1}}-u, v\right), \quad \forall v \in V_{h_{l}}^{0}\left(\Omega_{l}\right) .
\end{aligned}
$$

Since $\left.\left(u^{w, h_{l}}-P_{h_{l}} u\right)\right|_{\Omega_{l}} \in V_{h_{l}}\left(\Omega_{l}\right)$ and $V_{0}^{h_{l}}\left(\Omega_{l}\right) \subset V_{h_{l}}^{0}\left(\Omega_{l}\right)$, from the above formula and Lemma 3, we deduce that

$$
\left\|u^{w, h_{l}}-P_{h_{l}} u\right\|_{a, D} \leq\left\|u^{w, h_{l}}-P_{h_{l}} u\right\|_{b, \Omega_{l}}+\left|\lambda^{w, h_{l-1}}-\lambda\right|+\left\|u^{w, h_{l}}-u\right\|_{b, \Omega_{l}}
$$

By calculation, we have

$$
\begin{aligned}
\left\|u^{w, h_{l}}-P_{h_{l}} u\right\|_{b, \Omega_{l}} & \leq\left\|u^{w, h_{l-1}}-P_{h_{l}} u\right\|_{b, \Omega_{l}}+\left\|e^{h_{l}}\right\|_{b, \Omega_{l}} \\
& \leq\left\|u-P_{h_{l}} u\right\|_{b, \Omega_{l}}+\left\|u-u^{w, h_{l-1}}\right\|_{b, \Omega_{l}}+\left\|e^{h_{l}}\right\|_{b, \Omega_{l}} .
\end{aligned}
$$

Substituting the above relation in (64), we obtain

$$
\begin{aligned}
\left\|u^{w, h_{l}}-P_{h_{l}} u\right\|_{a, D} \leq & \left|\lambda^{w, h_{l-1}}-\lambda\right|+\left\|u^{w, h_{l-1}}-u\right\|_{b, \Omega_{l}} \\
& +\left\|u-P_{h_{l}} u\right\|_{b, \Omega_{l}}+\left\|e^{h_{l}}\right\|_{b, \Omega_{l}} .
\end{aligned}
$$

To estimate $\left\|e^{h_{l}}\right\|_{b, \Omega}$, we use the Aubin-Nitsche duality argument. For any given $f \in L_{2}\left(\Omega_{l}\right)$, consider the boundary value problem; find $\varphi \in H_{\Gamma}^{1}\left(\Omega_{l}\right):=\left\{v \in H_{\omega}^{1}\left(\Omega_{l}\right):\left.v\right|_{\partial \Omega_{l}} \mid\right.$ $\{-1\}=0\}$ such that

$$
a(\varphi, v)=b(f, v), \quad \forall v \in H_{\Gamma}^{1}\left(\Omega_{l}\right) .
$$

Let $\varphi$ be the generalized solution of (67) and $\varphi_{h_{1}}$ and $\varphi_{h_{l-1}}$ be finite element solutions of (67) in $V_{h_{l}}^{0}\left(\Omega_{l}\right)$ and $V_{h_{l-1}}^{0}\left(\Omega_{l}\right)$, respectively. Then,

$$
\begin{gathered}
\left\|\varphi-\varphi_{h_{l}}\right\|_{a, \Omega_{l}} \leq h_{l}^{\gamma}\|f\|_{b, \Omega_{l}}, \\
\left\|\varphi-\varphi_{h_{l-1}}\right\|_{a, \Omega_{l}} \leq h_{l-1}^{\gamma}\|f\|_{b, \Omega_{l}} .
\end{gathered}
$$

From (47) and (48), we obtain

$$
a\left(u^{w, h_{l}}, \varphi_{h_{l}}\right)=\lambda^{w, h_{l-1}} b\left(u^{w, h_{l-1}}, \varphi_{h_{l}}\right) .
$$

Then, by the definitions of $\varphi, \varphi_{h_{l}}$, and $e^{h_{l}}$, we deduce that

$$
\begin{aligned}
b\left(e^{h_{l}}, f\right)= & a\left(e^{h_{l}}, \varphi\right)=a\left(e^{h_{l}}, \varphi_{h_{l}}\right)=a\left(u^{w, h_{l}}-u^{w, h_{l-1}}, \varphi_{h_{l}}\right) \\
= & a\left(P_{h_{l}} u-u^{w, h_{l-1}}, \varphi_{h_{l}}\right)+a\left(u^{w, h_{l}}, \varphi_{h_{l}}\right)-a\left(P_{h_{l}} u, \varphi_{h_{l}}\right) \\
= & a\left(P_{h_{l}} u-u^{w, h_{l-1}}, \varphi_{h_{l}}\right)+\lambda^{w, h_{l-1}} b\left(u^{w, h_{l-1}}, \varphi_{h_{l}}\right) \\
& -\lambda b\left(u, \varphi_{h_{l}}\right) \\
= & a\left(P_{h_{l}} u-u^{w, h_{l-1}}, \varphi_{h_{l}}-\varphi\right)+a\left(P_{h_{l}} u-u^{w, h_{l-1}}, \varphi-\varphi_{h_{l-1}}\right) \\
& +a\left(P_{h_{l}} u-u^{w, h_{l-1}}, \varphi_{h_{l-1}}\right)+\lambda^{w, h_{l-1}} b\left(u^{w, h_{l-1}}, \varphi_{h_{l}}\right) \\
& -\lambda b\left(u, \varphi_{h_{l}}\right) \\
\leq & h_{l-1}^{\gamma}\left\|P_{h_{l}} u-u^{w, h_{l-1}}\right\|_{a, \Omega_{l}}\|f\|_{0, \Omega_{l}}+a\left(P_{h_{l}} u-u^{w, h_{l-1}}, \varphi_{h_{l-1}}\right) \\
& +\lambda^{w, h_{l-1}} b\left(u^{w, h_{l-1}}, \varphi_{h_{l}}\right)-\lambda b\left(u, \varphi_{h_{l}}\right) .
\end{aligned}
$$

Step 2 of Scheme 2 shows that

$$
a\left(u^{w, h_{0}}, \varphi_{h_{0}}\right)=\lambda^{w, h_{-1}} b\left(u^{w, h_{-1}}, \varphi_{h_{0}}\right),
$$

namely, for $l=1$,

$$
a\left(u^{w, h_{l-1}}, \varphi_{h_{l-1}}\right)=\lambda^{w, h_{l-2}} b\left(u^{w, h_{l-2}}, \varphi_{h_{l-1}}\right),
$$

for $l>1$, the above formula follows from (47) and (48). Therefore,

$$
\begin{aligned}
a\left(P_{h_{l}} u-u^{w, h_{l-1}}, \varphi_{h_{l-1}}\right) & =a\left(u-u^{w, h_{l-1}}, \varphi_{h_{l-1}}\right) \\
& =\lambda b\left(u, \varphi_{h_{l-1}}\right)-a\left(u^{w, h_{l-1}}, \varphi_{h_{l-1}}\right) \\
& =\lambda b\left(u, \varphi_{h_{l-1}}\right)-\lambda^{w, h_{l-2}} b\left(u^{w, h_{l-2}}, \varphi_{h_{l-1}}\right) \\
& \leqslant\left\|\lambda u-\lambda^{w, h_{l-2}} u^{w, h_{l-2}}\right\|_{b, \Omega_{l}}\|f\|_{b, \Omega_{l}} .
\end{aligned}
$$

It is clear that

$$
\begin{gathered}
\left|\lambda^{w, h_{l-1}} b\left(u^{w, h_{l-1}}, \varphi_{h_{l}}\right)-\lambda b\left(u, \varphi_{h_{l}}\right)\right| \lesssim\left\|\lambda^{w, h_{l-1}} u^{w, h_{l-1}}-\lambda u\right\|_{b, \Omega_{l}} \\
\|f\|_{b, \Omega_{l}} .
\end{gathered}
$$

Substituting the above two formulae in (70), we derive 


$$
\begin{aligned}
\left|b\left(e^{h_{l}}, f\right)\right| \lesssim & \left(h_{l-1}^{\gamma}\left\|P_{h_{l}} u-u^{w, h_{l-1}}\right\|_{a, \Omega_{l}}+\left\|\lambda u-\lambda^{w, h_{l-2}} u^{w, h_{l-2}}\right\|_{b, \Omega_{l}}\right. \\
& \left.+\left\|\lambda^{w, h_{l-1}} u^{w, h_{l-1}}-\lambda u\right\|_{b}\right)\|f\|_{b, \Omega_{l}} .
\end{aligned}
$$

Thus, we obtain

$$
\begin{aligned}
\left\|e^{h_{l}}\right\|_{a, \Omega_{l}} \lesssim & h_{l-1}^{\gamma}\left\|P_{h_{l}} u-u^{w, h_{l-1}}\right\|_{a, \Omega_{l}}+\left\|\lambda u-\lambda^{w, h_{l-2}} u^{w, h_{l-2}}\right\|_{b, \Omega_{l}} \\
& +\left\|\lambda^{w, h_{l-1}} u^{w, h_{l-1}}-\lambda u\right\|_{b} .
\end{aligned}
$$

Substituting (76) in (66), we obtain

$$
\begin{aligned}
\left\|u^{w, h_{l}}-P_{h_{l}} u\right\|_{a, D} \lesssim & \left\|u-P_{h_{l}} u\right\|_{b, \Omega_{l}}+h_{l-1}^{\gamma}\left\|P_{h_{l}} u-u^{w, h_{l-1}}\right\|_{a, \Omega_{l}} \\
& +\left\|\lambda u-\lambda^{w, h_{l-2}} u^{w, h_{l-2}}\right\|_{b, \Omega_{l}}+\left|\lambda^{w, h_{l-1}}-\lambda\right| \\
& +\left\|u^{w, h_{l-1}}-u\right\|_{b}
\end{aligned}
$$

Similarly, since $\left(G_{l} / \bar{D}\right) \subset \subset \Omega_{l}$, we deduce

$$
\begin{aligned}
\left\|u^{w, h_{l}}-P_{h_{l}} u\right\|_{a, D} \leq & \left\|u-P_{h_{l}} u\right\|_{b, \Omega_{l}}+h_{l-1}^{\gamma}\left\|P_{h_{l}} u-u^{w, h_{l-1}}\right\|_{a, \Omega_{l}} \\
& +\left\|\lambda u-\lambda^{w, h_{l-2}} u^{w, h_{l-2}}\right\|_{b, \Omega_{l}}+\left|\lambda^{w, h_{l-1}}-\lambda\right| \\
& +\left\|u^{w, h_{l-1}}-u\right\|_{b}
\end{aligned}
$$

The remainder is to analyze $\left\|u^{w, h_{l}}-P_{h_{l}} u\right\|_{a, \Omega \backslash \bar{G}}$. From (48), we see that

$$
\left\|u^{w, h_{l}}-P_{h_{l}} u\right\|_{a, \Omega \backslash \bar{\Omega}_{l}}=\left\|u^{w, h_{l-1}}-P_{h_{l}} u\right\|_{a, \Omega \mid \bar{\Omega}_{l}},
$$

which leads to

$$
\begin{aligned}
& \left\|u^{w, h_{l}}-P_{h_{l}} u\right\|_{a, \Omega \backslash \overline{G_{l}}} \\
& \leq\left\|u^{w, h_{l}}-P_{h_{l}} u\right\|_{a, \Omega \backslash \bar{\Omega}_{l}}+\left\|u^{w, h_{l-1}}-P_{h_{l}} u\right\|_{a, \Omega_{l} \bar{G}_{l}}+\left\|e^{h_{l}}\right\|_{a, \Omega_{l} \backslash \overline{G_{l}}} \\
& \leq\left\|u^{w, h_{l-1}}-P_{h_{l}} u\right\|_{a, \Omega \backslash \overline{G_{l}}}+\left\|e^{h_{l}}\right\|_{\left.a, \Omega_{l}\right\rangle \overline{G_{l}}} .
\end{aligned}
$$

It follows from (7), (47), and (62) that

$$
\begin{aligned}
a\left(e^{h_{l}}, v\right)= & \lambda^{w, h_{l-1}} b\left(u^{w, h_{l-1}}, v\right)-a\left(u^{w, h_{l-1}}, v\right)-\lambda b(u, v)+a(u, v) \\
= & \left(\lambda^{w, h_{l-1}}-\lambda\right) b(u, v)+\lambda^{w, h_{l-1}} b\left(u^{w, h_{l-1}}-u, v\right) \\
& -a\left(u^{w, h_{l-1}}-u, v\right), \quad \forall v \in V_{h}^{0}\left(\Omega_{l}\right) .
\end{aligned}
$$

Then, by Lemma 3, we have

$$
\left\|e^{h_{l}}\right\|_{a, \Omega_{l} / \bar{G}_{l}} \leqslant\left\|e^{h_{l}}\right\|_{b, \Omega_{l} / \bar{F}}+\left|\lambda^{w, h_{l-1}}-\lambda\right|+\left\|u^{w, h_{l-1}}-u\right\|_{a, \Omega_{l} / \bar{F}}
$$

where $F \subset \Omega$ satisfies $D \subset F \subset \subset G_{l}$. Substituting (82) in (80), we obtain

$$
\begin{aligned}
\left\|u^{w, h_{l}}-P_{h_{l}} u\right\|_{a, \Omega / \bar{G}_{l}} \lesssim & \left\|u^{w, h_{l-1}}-P_{h_{l}} u\right\|_{a, \Omega / \bar{G}_{l}}+\left\|e^{h_{l}}\right\|_{b, \Omega / \bar{F}} \\
& +\left|\lambda^{w, h_{l-1}}-\lambda\right|+\left\|u^{w, h_{l-1}}-u\right\|_{a, \Omega / \bar{F}}
\end{aligned}
$$

It follows from substituting (76) in the above inequality that

$$
\begin{aligned}
\left\|u^{w, h_{l}}-P_{h_{l}} u\right\|_{a, \Omega / \bar{G}_{l}} \leqslant & \left\|u^{w, h_{l-1}}-P_{h_{l}} u\right\|_{a, \Omega / \bar{G}_{l}}+h_{l-1}^{\gamma}\left\|P_{h_{l}} u-u^{w, h_{l-1}}\right\|_{a, \Omega_{l}} \\
& +\left\|\lambda u-\lambda^{w, h_{l-2}} u^{w, h_{l-2}}\right\|_{b, \Omega_{l}}+\left\|\lambda^{w, h_{l-1}} u^{w, h_{l-1}}-\lambda u\right\|_{b} \\
& +\left|\lambda^{w, h_{l-1}}-\lambda\right|+\left\|u^{w, h_{l-1}}-u\right\|_{b, \Omega / \bar{F}}
\end{aligned}
$$

Combining (60), (77), (78), and (84), finally, we obtain (59).

Theorem 3. Assume that the conditions of Theorem 1 hold. Then, there exists $u \in M(\lambda)$ such that

$$
\begin{gathered}
\left\|u^{w, h_{1}}-u\right\|_{a, \Omega} \lesssim h_{1}^{r+s-1}+w^{r}+H^{r+s-1+\gamma}, \\
\left\|u^{w, h_{1}}-u\right\|_{b, \Omega} \lesssim w^{r}+H^{r+s-1+\gamma}, \\
\left\|u^{w, h_{1}}-u\right\|_{a, \Omega / \bar{F}} \lesssim w^{r}+H^{r+s-1+\gamma}, \\
\left|\lambda^{w, h_{1}}-\lambda\right| \lesssim h_{1}^{2 r+2 s-2}+w^{2 r}+H^{2 r+2 s-2+2 \gamma} .
\end{gathered}
$$

Proof. Let $u \in M(\lambda)$ such that $u-u_{H}$ satisfies Lemma 2 . From Theorem 2, we know $l=1, h_{-1}=H, h_{0}=w$, $u^{w, h_{0}}=u^{w}, \lambda^{w, h_{0}}=\lambda^{w}, u^{w, h_{-1}}=u_{H}$, and $\lambda^{w, h_{-1}}=\lambda_{H}$; thus, we obtain

$$
\begin{aligned}
\left\|u^{w, h_{1}}-P_{h_{1}} u\right\|_{a, \Omega} \leq & \left\|u-P_{h_{1}} u\right\|_{0, \Omega_{1}}+w^{\gamma}\left\|P_{h_{1}} u-u^{w}\right\|_{a, \Omega_{1}} \\
& +\left\|\lambda u-\lambda_{H} u_{H}\right\|_{b, \Omega_{1}}+\left\|\lambda^{w} u^{w}-\lambda u\right\|_{b} \\
& +\left\|u^{w}-P_{h_{1}} u\right\|_{a, \Omega \backslash \overline{G_{1}}}+\left\|u^{w}-u\right\|_{a, \Omega_{1} \backslash \bar{F}} .
\end{aligned}
$$

Using Lemma 4, Theorem 1, and Lemma 2 to estimate the terms at the right-hand side of the above formula gives

$$
\begin{aligned}
\left\|u^{w, h_{1}}-P_{h_{1}} u\right\|_{a, \Omega} \leq & h_{1}^{r+s-1+\gamma}+w^{\gamma} w^{r+s-1}+H^{r+s-1+\gamma}+w^{r+s-1+\gamma} \\
& +\left(w^{r+s-1+\gamma}+w^{r}\right)+\left(w^{r+s-1+\gamma}+w^{r}\right) \\
\leq & H^{r+s-1+\gamma}+w^{r} .
\end{aligned}
$$

Combining (35) and (39) yields (85), (86), and (87). From (19), we have

$$
\lambda^{w, h_{1}}-\lambda=\frac{a\left(u^{w, h_{1}}-u, u^{w, h_{1}}-u\right)}{b\left(u^{w, h_{1}}, u^{w, h_{1}}\right)}-\lambda \frac{b\left(u^{w, h_{1}}-u, u^{w, h_{1}}-u\right)}{b\left(u^{w, h_{1}}, u^{w, h_{1}}\right)} .
$$


TABLE $1: V=-(1 / r)$

\begin{tabular}{lcccccrc}
\hline $\mathrm{DOF}_{H}$ & $\mathrm{DOF}_{w}$ & $\lambda_{H}$ & $\lambda^{w}$ & $\lambda^{w, h_{1}}$ & $\lambda^{w, h_{2}}$ & $\lambda^{w, h_{3}}$ & $\lambda^{w, h_{4}}$ \\
\hline 4 & 16 & -0.3558419 & -0.4662707 & -0.4903221 & -0.4963517 & -0.4975584 & -0.4976819 \\
8 & 64 & -0.4320788 & -0.4974509 & -0.4993601 & -0.4997939 & -0.4998737 & -0.4998816 \\
16 & 256 & -0.4735824 & -0.4998515 & -0.4999630 & -0.4999897 & -0.4999946 & -0.4999951 \\
32 & 1024 & -0.4918086 & -0.4999910 & -0.4999978 & -0.4999994 & -0.4999997 & -0.4999997 \\
\hline 4 & 16 & -0.10798797 & -0.12015585 & -0.12343262 & -0.12435602 & -0.12457107 & -0.12462204 \\
8 & 64 & -0.11060517 & -0.12445755 & -0.12476411 & -0.12484341 & -0.12486019 & -0.12486334 \\
16 & 256 & -0.12023285 & -0.12497717 & -0.12499349 & -0.12499748 & -0.12499831 & -0.12499849 \\
32 & 1024 & -0.12372913 & -0.12499867 & -0.12499966 & -0.12499990 & -0.12499995 & -0.12499996 \\
\hline
\end{tabular}

TABle 2: $V=\left(r^{2} / 2\right)$.

\begin{tabular}{lccccccc}
\hline $\mathrm{DOF}_{H}$ & $\mathrm{DOF}_{w}$ & $\lambda_{H}$ & $\lambda^{w}$ & $\lambda^{w, h_{1}}$ & $\lambda^{w, h_{2}}$ & $\lambda^{w, h_{3}}$ \\
\hline 4 & 16 & 2.3568087 & 1.7807519 & 1.5673755 & 1.5176295 & 1.5145651 \\
8 & 64 & 1.6871072 & 1.5041081 & 1.5010234 & 1.5002569 & 1.5001762 & 1.5142457 \\
16 & 256 & 1.5588758 & 1.5002540 & 1.5000634 & 1.5000159 & 1.5000109 & 1.5001727 \\
32 & 1024 & 1.5155354 & 1.5000157 & 1.5000039 & 1.5000010 & 1.5000007 & 1.5000007 \\
\hline 4 & 16 & 3.0744959 & 2.6046912 & 2.5284162 & 2.5069841 & 2.5039841 \\
8 & 64 & 3.0722770 & 2.5418424 & 2.5142338 & 2.5054025 & 2.5045811 & 2.5036625 \\
16 & 256 & 2.5890816 & 2.5004065 & 2.5001018 & 2.5000260 & 2.5000149 & 2.5045358 \\
32 & 1024 & 2.5245263 & 2.5000247 & 2.5000062 & 2.5000016 & 2.5000009 & 2.50000008 \\
\hline
\end{tabular}

Combining (85), (86), and (91) yields (88).

Theorem 4. Under the conditions of Theorem 1, we further assume that $R\left(\Omega_{i}\right)$ holds $(i=1,2, \ldots, l)$, and

$$
\begin{aligned}
w^{r} & =\mathcal{O}\left(H^{r+s-1+\gamma}\right), \\
h_{l}^{r+s-1} & \geq H^{r+s-1+\gamma} .
\end{aligned}
$$

Then, there exists $u \in M(\lambda)$ such that

$$
\begin{aligned}
\left\|u^{w, h_{l}}-u\right\|_{a, \Omega} & \leqslant h_{l}^{r+s-1}, \\
\left\|u^{w, h_{l}}-u\right\|_{b, \Omega} & \leqslant H^{r+s-1+\gamma}, \\
\left\|u^{w, h_{l}}-u\right\|_{a, \Omega \backslash \bar{F}} & \leqslant H^{r+s-1+\gamma}, \\
\left|\lambda^{w, h_{l}}-\lambda\right| & \leqslant h_{l}^{2 r+s+s-2} .
\end{aligned}
$$

Proof. Let $u \in M(\lambda)$ such that $u-u_{H}$ satisfies Lemma 2 . The proof of (93)-(96) is completed by induction. When $l=1$, Scheme 2 is actually Scheme 1 . Hence, from Theorems 1 and 3 and (92), we know that (93)-(96) hold for $l=0,1$.

Suppose (93)-(96) hold for $l-2$ and $l-1$, i.e.,

$$
\begin{aligned}
\left\|u^{w, h_{l-2}}-u\right\|_{a, \Omega} & \lesssim h_{l-2}^{r+s-1}, \\
\left\|u^{w, h_{l-2}}-u\right\|_{b, \Omega} & \lesssim H^{r+s-1+\gamma}, \\
\left\|u^{w, h_{l-2}}-u\right\|_{a, \Omega \backslash \bar{F}} & \lesssim H^{r+s-1+\gamma}, \\
\left|\lambda^{w, h_{l-2}}-\lambda\right| & \lesssim h_{l-2}^{2 r+s+s-2}, \\
\left\|u^{w, h_{l-1}}-u\right\|_{a, \Omega} & \lesssim h_{l-1}^{r+s-1}, \\
\left\|u^{w, h_{l-1}}-u\right\|_{b, \Omega} & \lesssim H^{r+s-1+\gamma}, \\
\left\|u^{w, h_{l-1}}-u\right\|_{a, \Omega \backslash \bar{F}} & \lesssim H^{r+s-1+\gamma}, \\
\left|\lambda^{w, h_{l-1}}-\lambda\right| & \lesssim h_{l-1}^{2 r+s+s-2} .
\end{aligned}
$$

Next, we shall prove that (93)-(96) hold for $l$. Using the above formula and Lemma 4 to estimate the terms at the right-hand side of (59) gives

$$
\begin{aligned}
\left\|u^{w, h_{l}}-P_{h_{l}} u\right\|_{a, \Omega} \lesssim & h_{l}^{r+s-1+\gamma}+h_{l-1}^{\gamma}\left(h_{l}^{r+s-1}+h_{l-1}^{r+s-1}\right)+H^{r+s-1+\gamma} \\
& +H^{r+s-1+\gamma}+\left(H^{r+s-1+\gamma}+h_{l}^{r}\right)+H^{r+s-1+\gamma} \\
\lesssim & H^{r+s-1+\gamma} .
\end{aligned}
$$

The combination of (35), (39), and (98) yields (93), (94), and (95). From (19), we have 
TABle 3: $V=r$.

\begin{tabular}{|c|c|c|c|c|c|c|c|}
\hline $\mathrm{DOF}_{H}$ & $\mathrm{DOF}_{w}$ & $\lambda_{H}$ & $\lambda^{w}$ & $\lambda^{w, h_{1}}$ & $\lambda^{w, h_{2}}$ & $\lambda^{w, h_{3}}$ & $\lambda^{w, h_{4}}$ \\
\hline 4 & 16 & 2.2305059 & 1.9209211 & 1.8729501 & 1.8611124 & 1.8601497 & 1.8600937 \\
\hline 8 & 64 & 1.9785415 & 1.8584870 & 1.8564450 & 1.8559369 & 1.8558894 & 1.8558874 \\
\hline 16 & 256 & 1.8939159 & 1.8559226 & 1.8557987 & 1.8557678 & 1.8557648 & 1.8557646 \\
\hline 32 & 1024 & 1.8658088 & 1.8557673 & 1.8557596 & 1.8557577 & 1.8557575 & 1.8557575 \\
\hline 4 & 16 & 2.9297073 & 2.7099046 & 2.6801814 & 2.6714983 & 2.6700907 & 2.6699643 \\
\hline 8 & 64 & 2.8582383 & 2.6735285 & 2.6699339 & 2.6688537 & 2.6686711 & 2.6686594 \\
\hline 16 & 256 & 2.7093909 & 2.6680156 & 2.6678766 & 2.6678430 & 2.6678375 & 2.6678370 \\
\hline 32 & 1024 & 2.6792284 & 2.6678409 & 2.6678323 & 2.6678303 & 2.6678299 & 2.6678299 \\
\hline
\end{tabular}
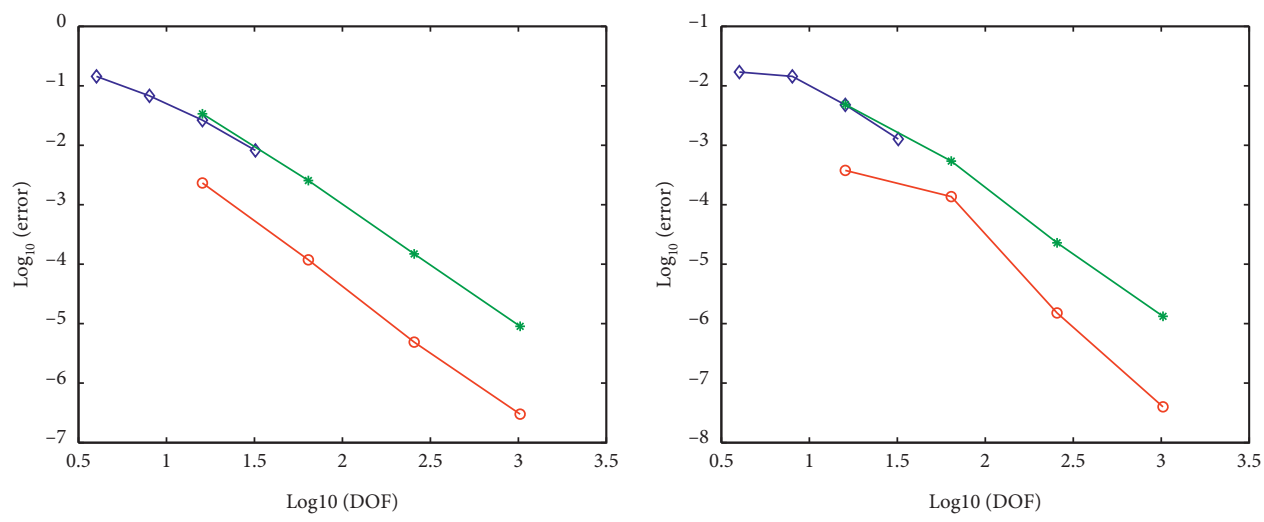

$$
\begin{aligned}
& \rightarrow\left|\lambda_{H}-\lambda\right| \\
& \rightarrow *\left|\lambda^{w}-\lambda\right| \\
& -\quad\left|\lambda^{w, h_{4}}-\lambda\right|
\end{aligned}
$$

(a)

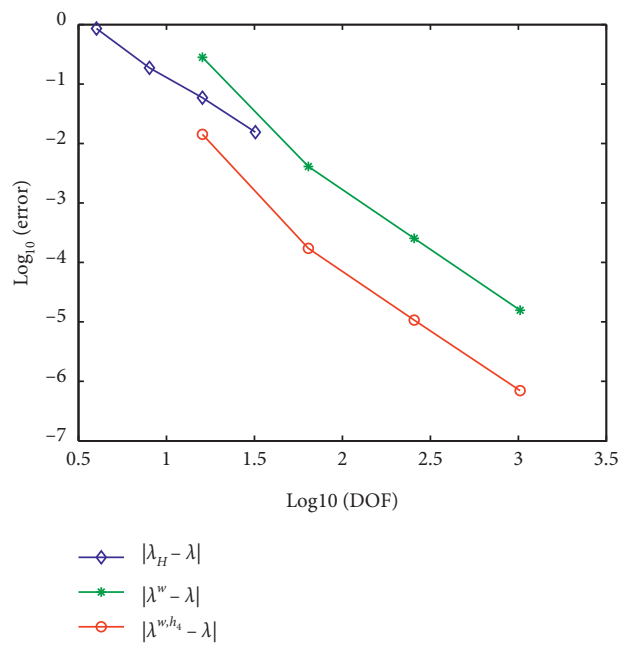

$$
\begin{aligned}
& \multimap\left|\lambda_{H}-\lambda\right| \\
& \rightarrow-\left|\lambda^{w}-\lambda\right| \\
& -\left|\lambda^{w, h_{4}}-\lambda\right|
\end{aligned}
$$

(b)

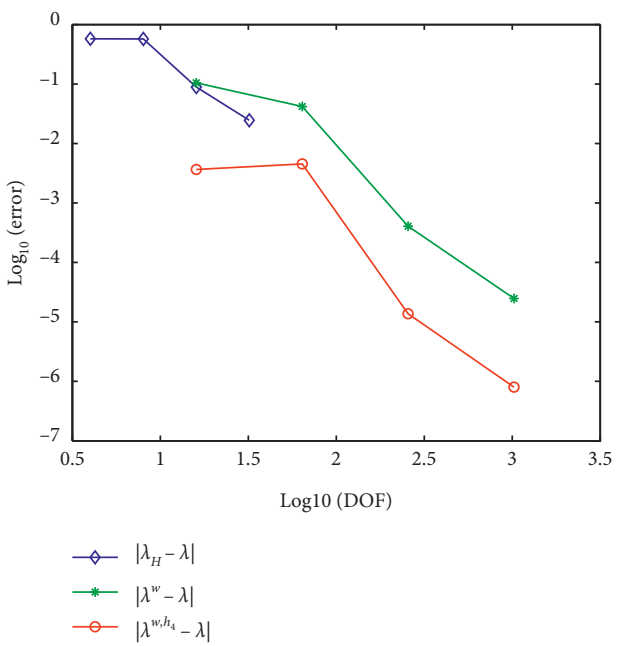

(d)

Figure 2: Continued. 


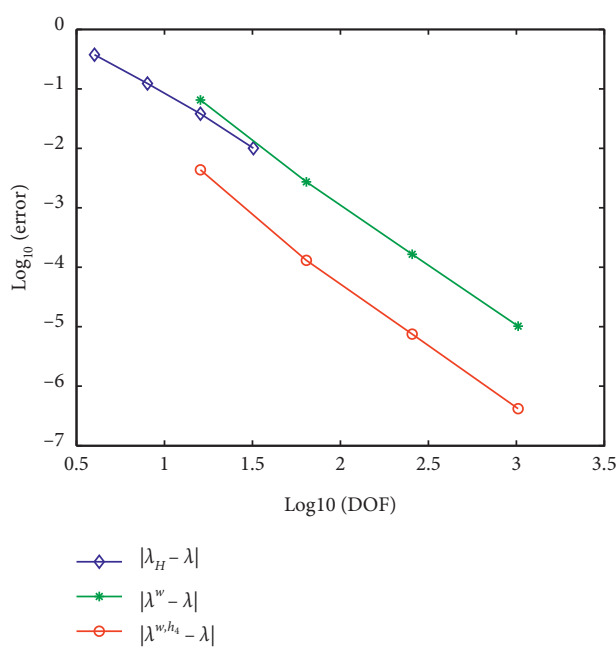

(e)

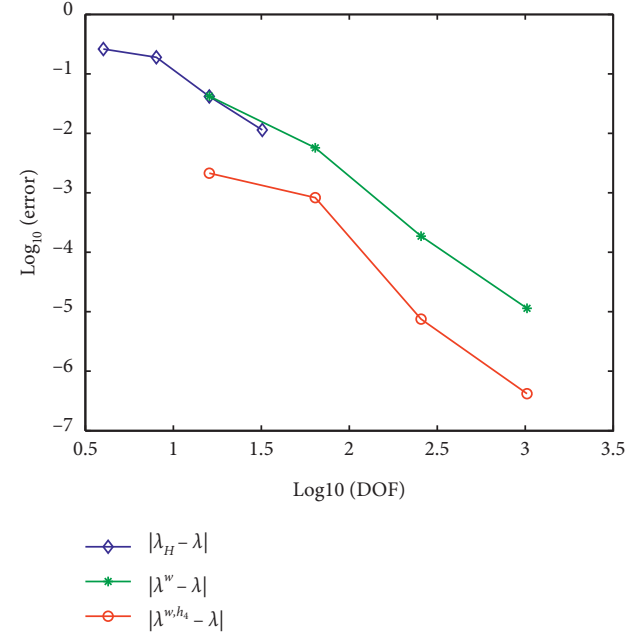

(f)

Figure 2: Error curve of numerical eigenvalues obtained by multiscale Scheme 2. (a) $V=-(1 / r), \lambda=-0.5$. (b) $V=-(1 / r), \lambda=-0.125$. (c) $V=-\left(r^{2} / 2\right), \lambda=1.5$. (d) $V=\left(r^{2} / 2\right), \lambda=2.5$. (e) $V=r, \lambda=1.855757081489$. (f) $V=r, \lambda=2.667829482852$.

$$
\lambda^{w, h_{l}}-\lambda=\frac{a\left(u^{w, h_{l}}-u, u^{w, h_{l}}-u\right)}{b\left(u^{w, h_{l}}, u^{w, h_{l}}\right)}-\lambda \frac{b\left(u^{w, h_{l}}-u, u^{w, h_{l}}-u\right)}{b\left(u^{w, h_{l}}, u^{w, h_{l}}\right)} .
$$

The combination of (93), (94), and (99) yields (96).

\section{Numerical Experiments}

We will report some numerical experiments by using linear finite element and quadratic spectral element on uniform meshes. In our numerical experiments, we use Scheme 2 to solve the problem such that $\Omega_{i}=\left(-1,-1+\left(1 / 2^{i}\right) \times(3 / 2)\right)$, $i=0,1,2, \ldots$, and locally fine grids have the same degree of freedom as that of globally mesoscopic grid (see Tables 1-3).

In our experiments, the parameter $\mu$ is taken to be 1 . We set $R=40$ for the eigenvalue problem with $V=-(1 / r)$ and $l=1$ and $R=15$ for the other cases. The coarse mesh size and the mesoscopic mesh size satisfy $\omega=H^{2}$ which means $\mathrm{DOF}_{w}=\mathrm{DOF}_{H}^{2}$.

We use MATLAB 2011b under the package of Chen (see [34]) to solve the problem, and the numerical results are shown in Tables 1-3. This tables corresponds to the results of different potential energy $V$. From these tables, we can see that, without increasing degree of freedom on locally fine grids, the first local defect correction can largely improve the accuracy of the eigenvalue, and the local defect corrections that follows can gradually improve the accuracy of the eigenvalue by overcoming the singularity at the origin. Here, we set

$$
\mathrm{DOF}_{w}=\mathrm{DOF}_{\Omega_{i}}, \quad i=1,2, \ldots
$$

In Figure 2, we also plot the error curve of numerical eigenvalues obtained by multiscale Scheme 2 . It can be seen that using coarse finite element space with the mesh size $H$, the error of the finite element eigenvalues is around poor accuracy $10^{-2}$. After performing the two-grid iteration, the error of the finite element eigenvalues can be increased from $10^{-2}$ to $10^{-5}$. The final mutiscale iteration can improve the error up to $10^{-6} \sim 10^{-7}$. These figures show the accuracy and effectiveness of our numerical scheme.

\section{Conclusion}

In this paper, we developed a efficient multiscale finite element method for solving the Schrödinger eigenvalue problem with three-dimensional domain. Our scheme can correct the defects repeatedly on the local refinement grid, which can solve the singularity problem efficiently. The error estimates of eigenvalues and eigenfunctions are proved. Some numerical examples are presented to verify the effectiveness of our numerical method.

\section{Data Availability}

The data used to support the findings of this study are included within the article.

\section{Conflicts of Interest}

The authors declare that they have no conflicts of interest.

\section{Acknowledgments}

The research was supported by NSFC (nos. 62062018, 11901135, and 11961009), Guizhou Province University Science and Technology top talents project (no. KY[2018] 047), Guizhou Key Laboratory of Big Data Statistics Analysis (no. BDSA20200102), and foundation of Guizhou Science and Technology Department (no. [2020]1Y015).

\section{References}

[1] D. B. Cook, Handbook of Computational Quantum Chemistry, Courier Dover Publications, New York, NY, USA, 2012. 
[2] Y. Saad, J. R. Chelikowsky, and S. M. Shontz, "Numerical methods for electronic structure calculations of materials," SIAM Review, vol. 52, no. 1, pp. 3-54, 2020.

[3] J. H. Bramble and J. E. Osborn, "Rate of convergence estimates for nonselfadjoint eigenvalue approximations," Mathematics of Computation, vol. 27, no. 123, p. 525, 1973.

[4] J. Han and Y. Yang, "A class of spectral element methods and its A priori/A posteriori error estimates for 2nd-order elliptic eigenvalue problems," Abstract and Applied Analysis, vol. 2013, Article ID 262010, 14 pages, 2013.

[5] V. Heuveline and R. Rannacher, "A posteriori error control for finite element approximations of elliptic eigenvalue problems," Advances in Computational Mathematics, vol. 15, no. 1/4, pp. 107-138, 2001.

[6] V. Heuveline and R. Rannacher, "Adaptive FE eigenvalue approximation with application to hydrodynamic stability analysis," in Proceedings of the international conference on Advances in Numerical Mathematics, Moscow, Russia, September 2005.

[7] H. Bi, Y. Zhang, and Y. Yang, "Two-grid discretizations and a local finite element scheme for a non-selfadjoint Stekloff eigenvalue problem," Computers \& Mathematics with Applications, vol. 79, no. 7, pp. 1895-1913, 2020.

[8] K. Kolman, "A two-level method for nonsymmetric eigenvalue problems," Acta Mathematicae Applicatae Sinica, English Series, vol. 21, no. 1, pp. 1-12, 2005.

[9] J. Zhang and X. Yang, "Decoupled, non-iterative, and unconditionally energy stable large time stepping method for the three-phase Cahn-Hilliard phase-field model," Journal of Computational Physics, vol. 404, Article ID 109115, 2020.

[10] Y. Yang and X. Fan, "Generalized Rayleigh quotient and finite element two-grid discretization schemes," Science in China Series A: Mathematics, vol. 52, no. 9, pp. 1955-1972, 2009.

[11] Y. Yang, " $\$ \mathrm{H} 2 \$$-Conforming methods and two-grid discretizations for the elastic transmission eigenvalue problem," Communications in Computational Physics, vol. 28, no. 4, pp. 1366-1388, 2020.

[12] Q. Li and G. Du, "Local and parallel finite element methods based on two-grid discretizations for the nonstationary Navier-Stokes equations," Numerical Algorithms, pp. 1-22, 2021.

[13] T. Lü and Y. Feng, "Splitting extrapolation based on domain decomposition for finite element approximations," Science in China Series E: Technological Sciences, vol. 40, no. 2, pp. 144-155, 1997.

[14] J. Zhang and X. Yang, "Unconditionally energy stable large time stepping method for the L2-gradient flow based ternary phase-field model with precise nonlocal volume conservation," Computer Methods in Applied Mechanics and Engineering, vol. 361, Article ID 112743, 2020.

[15] Y. Yang, H. Bi, and S. Li, "The extrapolation of numerical eigenvalues by finite elements for differential operators," Applied Numerical Mathematics, vol. 69, pp. 59-72, 2013.

[16] J. Zhang and X. Yang, "A fully decoupled, linear and unconditionally energy stable numerical Scheme for a meltconvective phase-field dendritic solidification model," Computer Methods in Applied Mechanics and Engineering, vol. 363, Article ID 112779, 2020.

[17] J. Zhang, C. Chen, X. Yang, Y. Chu, and Z. Xia, "Efficient, Non-iterative, and second-order accurate numerical algorithms for the anisotropic Allen-Cahn Equation with precise nonlocal mass conservation," Journal of Computational and Applied Mathematics, vol. 363, pp. 444-463, 2020.
[18] J. C. Xu and A. H. Zhou, "Local and parallel finite element algorithms based on two-grid discretizations," Mathematics of Computation, vol. 69, pp. 881-909, 2000.

[19] Y. He, J. Xu, A. Zhou, and J. Li, "Local and parallel finite element algorithms for the Stokes problem," Numerische Mathematik, vol. 109, no. 3, pp. 415-434, 2008.

[20] Y. He, L. Mei, Y. Shang, and J. Cui, "Newton iterative parallel finite element algorithm for the steady Navier-Stokes equations," Journal of Scientific Computing, vol. 44, no. 1, pp. 92-106, 2010.

[21] J. Xu and A. Zhou, "Local and parallel finite element algorithms for eigenvalue problems," Acta Mathematicae Applicatae Sinica, English Series, vol. 18, no. 2, pp. 185-200, 2002.

[22] X. Dai and A. Zhou, "Three-scale finite element discretizations for quantum eigenvalue problems," SIAM Journal on $\mathrm{Nu}$ merical Analysis, vol. 46, no. 1, pp. 295-324, 2008.

[23] H. Bi, Y. Yang, and H. Li, "Local and parallel finite element discretizations for eigenvalue problems," SIAM Journal on Scientific Computing, vol. 35, no. 6, pp. A2575-A2597, 2013.

[24] Y. Yang and H. Bi, "Two-grid finite element discretization schemes based on shifted-inverse power method for elliptic eigenvalue problems," SIAM Journal on Numerical Analysis, vol. 49, no. 4, pp. 1602-1624, 2011.

[25] Y. Yang and J. Han, "Multilevel finite element discretizations based on local defect correction for nonsymmetric eigenvalue problems," Computers \& Mathematics with Applications, vol. 70, no. 8, pp. 1799-1816, 2015.

[26] A. Gordon, C. Jirauschek, and F. X. KRtner, "Numerical solver of the time-dependent Schrödinger equation with Coulomb singularities," Physical Review A, vol. 73, no. 4, p. 838, 2006.

[27] J. Zhang, F. Lin, and J. Wang, "An efficient spectral-Galerkin method based on a dimension reduction scheme for eigenvalue problems of Schrödinger equations," Mathematical Methods in the Applied Sciences, vol. 42, no. 6, pp. 2069-2082, 2019.

[28] J. Zhang and X. Fan, "An efficient spectral method for the helmholtz transmission eigenvalues in polar geometries," Discrete and Continuous Dynamical Systems, vol. 24, no. 9, pp. 4799-4813, 2019.

[29] J. An, "A Legendre-Galerkin spectral approximation and estimation of the index of refraction for transmission eigenvalues," Applied Numerical Mathematics, vol. 108, pp. 171-184, 2016.

[30] P. Grisvard, Elliptic Problems in Nonsmooth Domains, Pitman, London, UK, 1985.

[31] S. C. Brenner and L. R. Scott, The Mathematical Theory of Finite Element Methods, Springer-Verlag, New York, NY, USA, 2nd edition, 2002.

[32] I. Babuska and J. E. Osborn, "Eigenvalue problems," in Finite Element Methods (Part 1), Handbook of Numerical Analysis, P. G. Ciarlet and J. L. Lions, Eds., pp. 640-787, Elsevier Science Publishers, North-Holand, Netherlands, 1991.

[33] L. B. Wahlbin, "Local behavior in finite element methods," in Finite Element Methods (Part1), Handbook of Numerical Analysis, P. G. Ciarlet and J. L. Lions, Eds., Elsevier SciencePublishers, North-Holand, Netherlands, pp. 355-522, 1991.

[34] L. Chen, IFEM: An Innovative Finite Element Methods Package in MATLAB, International Federation for Emergency Medicine, Bengaluru, India, 2008. 